\title{
Les formes socioculturelles de la malnutrition des enfants de moins de cinq ans dans la commune de Karimama au Nord du Bénin
}

\author{
Edoun Emmanuel Guy, (Doctorant) \\ Mongbo L. Roch, (Professeur Titulaire)
}

École Doctorale Pluridisciplinaire (EDP), Laboratoire d'Analyse des

Dynamiques Sociales et des Etudes du Développement (LADYD),

Université d'Abomey-Calavi (UAC), Bénin

Doi:10.19044/esj.2020.v16n16p73 ～URL:http://dx.doi.org/10.19044/esj.2020.v16n16p73

\section{Résumé}

Un bon état nutritionnel est le gage d'une vie saine et active. Cette réalité est encore un rêve dans la commune de Karimama, milieu où la malnutrition sous toutes ses formes a pris des proportions intolérables. Cette étude socio-anthropologique a pour objectif d'identifier les facteurs socioculturels qui servent de ferment à la malnutrition des enfants de moins de cinq ans. A partir d'une méthode d'observation et d'analyse croisée prenant en compte des faits et gestes jusque-là volontairement délaissés parce que considérés comme mineurs, anodins ou accessoires, il ressort que les pratiques socioculturelles ainsi que certains mécanismes d'influence ont un rôle important dans l'état nutritionnel des enfants. Ainsi, les variables liées aux croyances, aux mariages précoces et forcés, au pouvoir de décision des femmes et à la discrimination sexuelle des femmes se présentent comme le soubassement de la dénutrition des enfants de moins de cinq ans dans la commune. La prise en compte de l'environnement socioculturel et l'intégration des variables qui lui sont liées dans les actions essentielles en nutrition contribueraient à réduire sensiblement la persistance de la malnutrition des enfants de moins de cinq ans.

Mots clés : Formes socioculturelles, malnutrition, dénutrition, enfants de moins de cinq ans et Bénin 


\title{
Sociocultural Forms of Malnutrition of Children Under Five Years Old in the Commune of Karimama
}

\author{
Edoun Emmanuel Guy, (Doctorant) \\ Mongbo L. Roch, (Professeur Titulaire)
}

École Doctorale Pluridisciplinaire (EDP), Laboratoire d'Analyse des

Dynamiques Sociales et des Etudes du Développement (LADYD),

Université d'Abomey-Calavi (UAC), Bénin

\begin{abstract}
The good nutritional status is the guarantee of a healthy and active life. This reality is still a dream in the commune of Karimama, an environment where malnutrition in all its forms has taken on intolerable proportions. The aim of this socio-anthropological study is to identify the socio-cultural factors which serve as a catalyst for malnutrition of children under the age of five years. From a method of observation and cross-analysis taking into account facts and gestures up to now deliberately neglected because considered as minor, trivial or incidental, it emerges that socio-cultural practices as well as certain mechanism of influence have important role in the nutritional status of children. Thus the variables linked to beliefs, early and forced marriages, the decision-making power of women and the sexual discrimination of women appear to be the basis of undernutrition of children under the age of five years in the commune. Taking into account the socio-cultural environment and integrating the variables linked to it in essential nutrition actions would help to significantly reduce the persistence of malnutrition of children under the age of five years.
\end{abstract}

Keywords: Socio-cultural forms, malnutrition, undernutrition, children under five years old and Benin

\section{Introduction}

La dénutrition et plus généralement la faim dans le monde sont des sujets qui touchent à des problèmes fondamentaux du monde moderne. À l'échelle de la planète, plus de 150 millions d'enfants accusent de retard de croissance et l'émaciation continue de toucher plus de 50 millions d'enfants (Rapport UNICEF, 2018, p2). Plus de 2 milliards de personnes présentent une carence en micronutriments (vitamine A, iode, fer et zinc). En Afrique, le nombre d'enfants souffrant de cette pathologie, porté par la croissance 
démographique, a régulièrement augmenté, passant de 50,6 millions en 2000 à 58,7 millions en 2017 (UNICEF, 2018, p2). Les progrès accomplis à ce jour ne sont tout simplement pas suffisants. Les ravages de la malnutrition s'étendent aussi à des millions de survivants qui restent infirmes, chroniquement vulnérables aux infections et intellectuellement diminués (C. Bellamy, 1998). La malnutrition a de sérieuses conséquences sur la santé et la qualité générale de vie. Les conséquences néfastes de la malnutrition dans les premières années de vie sont en fait souvent irréversibles (CG. Victora et al. 2008). L'aggravation du risque de décès des enfants liée à la malnutrition serait due à un affaiblissement du système immunitaire de l'enfant qui accroit sa vulnérabilité aux maladies infectieuses (D.L. Peletier \& al, 1993) cité par R. Ndamobissi (2017, p.120). De nombreuses études pluridisciplinaires ont montré aussi que le développement d'une nation est tributaire de l'état nutritionnel de sa population (F. Delpeuch et G. Salem, 2002, p.67). Le développement économique et social des pays est fonction de leur capacité à répondre aux besoins nutritionnels des femmes et des enfants (UNICEF, 1998, p.14). C'est sûrement pour cela qu'en 2012, au regard de la récession économique mondiale, la malnutrition a été placée sur l'agenda du G8 (les huit États industrialisés les plus riches du monde) comme une priorité pour le développement. Et en 2013, l'Assemblée générale de la santé s'est fixé d'atteindre d'ici 2025 une réduction de $40 \%$ (WHO, 2014) du nombre des enfants affectés par la malnutrition chronique, "pour sauver 70 millions d'enfants malnutris (Unicef, 2013, page iii) ». La malnutrition constitue une forme de synthèse des résultats obtenus dans la prise en charge des quatre dimensions de la sécurité alimentaire (disponibilité, accessibilité, stabilité et utilisation) et dans la gestion des interactions des facteurs qui y concourent (B. Soulé, 2013, p.7). Plusieurs facteurs sont à l'origine de la malnutrition. L'état de santé de l'enfant, l'alimentation, le niveau d'éducation de la mère, le niveau de vie du ménage, l'environnement, les politiques et programmes, etc. tiennent des rôles fondamentaux sur l'état nutritionnel des petits enfants. Selon A. Sen (1998), chez les enfants, la malnutrition désigne un ensemble de désordres biologiques caractérisés par un arrêt ou un retard de la croissance. Pour G. Beaton et al, (1990), la malnutrition résulte de l'interaction entre un apport alimentaire inadéquat et la maladie, et mène la plupart à des déficits anthropométriques observés chez les enfants. La malnutrition est aussi associée à la sécheresse ou à d'autres catastrophes naturelles. Ainsi, pour E. Nelson et al (2009, p.8) «le changement climatique anéantirait en grande partie l'amélioration des niveaux de malnutrition des enfants qui se serait produite sans changement climatique ». De façon plus précise, «les changements de saisonnalité influencent les moyens de subsistance, la production alimentaire, l'accès à l'eau, la morbidité, et enfin le pic saisonnier de la dénutrition » $(\mathrm{ACF}, 2008)$. Les causes profondes de la malnutrition et les 
facteurs qui la favorisent sont complexes et multifactoriels »(FAO, OMS, 2014, p.2). Elle résulte de la combinaison de facteurs individuels, familiaux, communautaires, nationaux et internationaux allant de la maladie, des croyances culturelles et coutumes et des taux élevés de fertilité aux faibles conditions économiques et à l'accès limité aux services de santé et sociaux (D.L. Pelletier, 1994, p.410). Les facteurs socioculturels auxquels on l'associe peu ou presque pas surtout dans les campagnes et les programmes de lutte contre la malnutrition constituent pourtant des facteurs structurants. En effet, « l'alimentation est à la fois hors et dans le fait social en ce sens que les mœurs alimentaires préexistent aux individus, mais que l'alimentation est plus biologique que sociologique avec pour conséquence, l'alimentation devient un lieu d'indexation de problématiques sociologiques plus fortes » (J. P. Poulain, 2002, p. 212). La commune de Karimama où les taux de malnutrition sont encore très élevés, $40,3 \%$ pour le retard de croissance, $13 \%$ pour l'émaciation et $30,8 \%$ pour l'insuffisance pondérale se doit de considérer dans les efforts de lutte tous les facteurs susceptibles de perpétuer cette « anomie ». Certes, la situation sociale de la commune, marquée par plusieurs décennies de catastrophes naturelles, de crises alimentaires et une pauvreté très aiguë, mérite une attention particulière. Mais ces taux très critiques de malnutrition sont très préoccupants pour une commune qui, depuis plusieurs années, bénéficie de nombreux programmes de nutrition et de lutte contre la pauvreté. Pourquoi la commune reste-t-elle affecter par la persistance d'une forte prévalence de la malnutrition des enfants en dépit des efforts de développement ? N'y-a-t-il pas des pratiques sociales, des normes culturelles qui aggravent les risques de malnutrition des enfants dans la commune ? Nous faisons l'hypothèse que la malnutrition persistante des enfants de moins de cinq ans dans la commune est occasionnée non seulement par le faible développement économique de la commune, le contexte de vulnérabilité alimentaire, des lacunes liées à la nutrition au niveau individuel, familial et communautaire mais aussi et surtout par un environnement socioculturel très austère vis-à-vis des enfants et des femmes. Le but de cette recherche est d'étudier les déterminants les plus subtils, même ceux qui ne s'inscrivent pas forcement dans ceux des experts, des spécialistes et des chercheurs en nutrition. Ainsi, se fixant pour objectif d'identifier les formes socioculturelles de la malnutrition des enfants de moins de cinq ans à Karimama, cet article essaye de rechercher les déterminants considérés comme mineurs, anodins ou accessoires mais qui influencent la nutrition et constituent un ferment pour la dénutrition des enfants de moins de cinq ans dans la commune. 


\section{Matériels et méthodes \\ 2.1. Matériels}

Plusieurs matériels ont été utilisés pour la réalisation de cette étude. Il s'agit entre autres des ressources humaines et financières, du matériel informatique, des fiches d'enquête et d'entretien, les fiches de suivi des animateurs PMASN. Les matériels informatiques utilisés sont entre autres : Un ordinateur, le tableur Excel 2013, logiciel STATA MP 13.

\subsection{Collecte de données}

L'étude a été conduite dans la Commune de Karimama située entre $12^{\circ} 4^{\prime} 0^{\prime \prime}$ ' latitude Nord et $3^{\circ} 10^{\prime} 60^{\prime \prime}$ longitude Est. Son altitude moyenne est de $200 \mathrm{~m}$ au-dessus du niveau de la mer dont elle est à plus de $773 \mathrm{~km}$ à vol d'oiseau. Le climat de la Commune est de type sahélo-soudanien marqué par une saison sèche de Novembre à Avril. La Commune se trouve dans la vallée du Niger qui va de Kargui jusqu'au Mékrou. Ces caractéristiques sont à la fois un atout et une contrainte pour la nutrition et la sécurité alimentaire des ménages. Nous avons mené une étude à la fois quantitative avec l'exploitation des données anthropométriques et qualitative utilisant des groupes de discussion et des entrevues individuelles semi-structurées. Les données ont été recueillies dans le cadre d'une recherche en thèse. Le niveau d'observation est le ménage. La méthode d'échantillonnage par grappes stratifiés à deux degrés a été utilisée et a permis d'enquêter 370 ménages dans quatorze (14) villages vulnérables repartis dans les cinq arrondissements que compte la commune. Ces villages ont été sélectionnés de manière raisonnée. Les villages ayant les effectifs importants d'enfants souffrant de malnutrition aigüe globale $(\mathrm{P} / \mathrm{T}<-$ $2 \mathrm{Z}$ - scores et/ou œdèmes; $\mathrm{PB}<125 \mathrm{~mm}$ et/ou Edèmes ; référence OMS, 2006) ont été choisis en se basant sur les données des projets PNC (Projet de Nutrition Communautaire) et PMASN (Projet Multisectoriel de l'Alimentation, de la Santé et de la Nutrition) exécutés dans la commune. Dans cette démarche qualitative, des données d'observation fine ont été jumelées à des données déclaratives émanant d'entretiens formels et informels, individuels au niveau des ménages et de groupe de discussion (focus group). Les données anthropométriques viennent de la collecte routinière de données pour le suivi des enfants du projet PMSAN. Le cadre conceptuel de l'UNICEF (1997) a servi de cadre de référence pour l'identification des causes de la malnutrition.

\subsection{Méthode d'Analyse}

Cette recherche est basée d'une part, sur une analyse quantitative et statistique des données anthropométriques de collecte routinière du projet PMASN pour le suivi des enfants et d'autre part sur une analyse qualitative des informations de perceptions et pratiques d'ordre sociologique au niveau 
communautaire de la nutrition des enfants. Ainsi, plusieurs méthodes dans une démarche d'analyse croisée et itérative ont permis d'analyser les données. La méthode quantitative basée sur l'analyse statistique des données anthropométriques a porté plus particulièrement sur l'impact des facteurs au niveau individuel, ménage et communautaire. L'analyse qualitative a permis d'identifier les facteurs socioculturels et nutritionnels auxquels la malnutrition est associée. A travers l'analyse du contenu des messages clefs issus des observations directes, des entretiens individuels et des discussions de groupes, il a été détecter les représentations et les différents « sens » que les acteurs donnent aux pratiques liées à l'alimentation des enfants. Enfin, l'analyse descriptive de régression linéaire multiple et de régression logistique a permis de vérifier la liaison ou l'association entre la variable dépendante et chacune des variables indépendantes. Le logiciel d'analyse statistique STATA a été utilisé pour ces analyses et a permis d'appréhender la relation entre l'état nutritionnel des enfants avec chacun des facteurs socio-économiques, socioculturels, sanitaires, alimentaires et nutritionnels (variables indépendantes du cadre théorique et conceptuel).

\section{Résultats}

\subsection{Niveaux et tendances de la malnutrition au Bénin}

Au Bénin, selon les résultats de l'Enquête Démographique et de Santé (EDS, 2017-2018), 32\% des enfants souffrent de malnutrition chronique et $11 \%$ en souffrent sous sa forme sévère. La prévalence du retard de croissance a tendance à augmenter avec l'âge jusqu'à 24-35 mois, groupe d'âges dans lequel elle atteint son niveau le plus élevé (41\%), puis elle diminue par la suite tout en demeurant à un niveau élevé, puisque entre 48 à 59 mois, un tiers des enfants (33\%) sont trop petits pour leur âge. L'insuffisance d'apports alimentaires est le plus souvent associée à un niveau de vie modeste. Parfois les ménages sont conscients du fait qu'ils doivent varier leur alimentation et consommer des aliments riches en fer mais, par manque de moyens ou parfois de connaissance, ils ne peuvent modifier leur comportement alimentaire et se contentent de consommer ce qui est disponible.

\subsection{Niveaux et tendances de la malnutrition dans la commune}

Il est constaté que les enfants du milieu rural sont davantage affectés par le retard de croissance que ceux du milieu urbain (36\% contre $28 \%$ ). Mais la situation de la commune rurale de Karimama est assez critique (tableau 1). 
Tableau 1: Évolution de la malnutrition de 2011 et 2018 à Karimama

\begin{tabular}{ccccc}
\hline Différentes formes de malnutrition & \multicolumn{2}{c}{ PNC } & CAP & PMASN \\
\cline { 2 - 5 } & 2011 & 2015 & 2016 & 2018 \\
\hline Malnutrition aigüe ou Emaciation \% & 22,92 & 16,7 & 15,1 & 13,0 \\
\hline Insuffisance pondérale \% & 47,92 & 38,0 & 32,9 & 30,8 \\
\hline Malnutrition chronique ou Retard de croissance \% & 64,58 & 43,3 & 40,2 & 40,3 \\
\hline
\end{tabular}

Source: Données PNC, 2015, Etude CAP et PMASN

Le taux de malnutrition chronique a évolué de $64,58 \%$ en 2011, 43,3 en 2015, $40,2 \%$ en 2016 à 40, 3\% en 2018. L'insuffisance pondérale est passée de $47,92 \%$ en 2011 à $30,8 \%$ en 2018 et l'émaciation dans la même période est passée de $22,92 \%$ à $13,0 \%$. Certes entre 2011 et 2018, ces différents taux de malnutrition ont considérablement baissé mais par rapport à la norme de l'OMS (2006) et comparé au niveau national, ces taux restent encore très élevés. Plusieurs facteurs sont invoqués pour être à l'origine de la malnutrition des enfants de moins de cinq ans dans la commune. Ces facteurs comprennent à la fois les déterminants socio-économiques au niveau communautaire et du ménage, les facteurs comportementaux de soins et des facteurs biomédicaux et sociodémographiques au niveau individuel (T. Black, et al, 2008). Partant de l'hypothèse de l'étude, le cadre adopté pour l'analyse des facteurs déterminants la malnutrition des enfants de moins de cinq ans dans la commune se base sur le cadre conceptuel de l'UNICEF (1997) et prend en compte les facteurs socioculturels associés à la malnutrition dans ce milieu.

\section{Déterminants de la malnutrition liés aux caractéristiques individuelles de l'enfant}

Il est retenu ici le sexe et l'âge de l'enfant qui sont des paramètres biologiques de différenciation du statut de l'enfant qui influencent son alimentation et son état de santé selon les valeurs et les croyances sociales.

\subsubsection{Malnutrition et sexe de l'enfant}

Que nous soyons en présence d'une malnutrition chronique, d'une insuffisance pondérale ou d'une émaciation, les taux varient selon que c'est une fille ou un garçon (tableau 2). Les filles ne souffrent pas de la malnutrition comme les garçons en souffrent.

Tableau 2: Type de malnutrition (taux \%) selon le sexe de l'enfant dans la commune

\begin{tabular}{llll}
\hline \multirow{2}{*}{ Sexe de l'enfant } & \multicolumn{3}{c}{ Type de malnutrition (taux \%) } \\
\cline { 2 - 4 } \multicolumn{1}{c}{ Masculin } & Taille/ Age <-2ET & Poids/Age <-2ET & Poids/Taille <-2ET \\
\hline Féminin & 39,7 & 37,2 & 18,9 \\
\hline Ensemble & 37,0 & 38,3 & 17,8 \\
\hline $\begin{array}{l}\text { Significativité. } \\
\text { p=probabilité du Chi2 }\end{array}$ & 0,006 & 37,8 & 18,4 \\
\hline
\end{tabular}

Source : PMSAN, $1^{\text {er }}$ trimestre 2018 
Le croisement du sexe de l'enfant avec les taux de malnutrition (Taille/ Age <-2ET ; Poids/Age <-2ET et Poids/Taille <-2ET) laisse apparaître que les filles de moins de 5 ans sont relativement moins nombreuses que les garçons à être malnutries quelque soit le type de malnutrition. Cette différenciation, même légère, entre les taux de malnutrition des deux sexes pourrait être expliquée sur le plan culturel par les pratiques d'allaitement, de sevrage et d'alimentation du jeune enfant. Les garçons seraient un peu plus "exigeants" et "gourmands" dans la prise des tétés et les mamans n'accèdent pas toujours à leur exigence.

\subsubsection{Malnutrition et groupes d'âge de l'enfant en mois}

Quelque soit le type de malnutrition, le taux augmente avec l'âge de l'enfant. Ce résultat confirme que l'âge de l'enfant est fortement corrélé à son état nutritionnel (tableau 3).

Tableau 3 : Type de malnutrition (taux \%) selon le groupe d'âge de l'enfant en mois

\begin{tabular}{|c|c|c|c|}
\hline \multirow{2}{*}{ Groupe d'âge } & \multicolumn{3}{|c|}{ Type de malnutrition (taux \%) } \\
\hline & $\begin{array}{l}\text { Taille/ Age } \\
\text { 2ET }\end{array}$ & $<-\quad$ Poids/Age $<-2$ ET & Poids/Taille <-2ET \\
\hline$<6$ mois & 5,1 & 7,00 & 13 \\
\hline 6-11 mois & 16,9 & 33,8 & 35,1 \\
\hline 12-23 mois & 45,2 & 50,0 & 33,2 \\
\hline 24-35 mois & 46,1 & 47,4 & 17,2 \\
\hline 36-47 mois & 47,2 & 37,0 & 12,0 \\
\hline 48-59 mois & 40,1 & 32,1 & 8,9 \\
\hline Total & 37,8 & 39,2 & 17,9 \\
\hline Significativité & 0,000 & 0,000 & 0,000 \\
\hline
\end{tabular}

Source : PMSAN, $1^{\text {er }}$ trimestre 2018

On remarque que de la naissance à 24 mois, le taux de malnutrition augmente. Cette tendance s'inverse rapidement après 24 mois pour l'insuffisance pondérale et l'émaciation. La tendance est la même à quelque différence près pour la malnutrition chronique après 24 mois. La nutrition de l'enfant est ainsi fonction de son âge. Mais dans la commune de Karimama, cela peut s'expliquer par le fait que les mères font très peu de différence entre nourrir un nourrisson de moins de 6 mois avec un enfant de 12 à 23 mois et ce dernier avec celui de plus de 36 mois. Dans la commune, une bouillie locale appelée "kommandi" à base de céréale sorgho sert à nourrir les enfants quelque soit leur âge et ceci tout au long de la journée. La pratique de l'allaitement maternel exclusif n'est guère répandue.

\subsubsection{Malnutrition, allaitement et aliments de complément}

Les taux de malnutrition différent selon que les enfants sont au sein ou nourris aux aliments de complément (Tableau 4). 
Tableau 4 : Taux de malnutrition des enfants selon l'allaitement et les aliments de complément

\begin{tabular}{llll}
\hline Selon l'alimentation & \multicolumn{3}{c}{ Type de malnutrition (taux \%) } \\
\hline & Taille/ Age $<-2 E T$ & Poids/Age <-2ET & Poids/Taille <-2ET \\
\hline Enfants au sein (0-6 mois) & 0,1 & 1,1 & 0,9 \\
\hline $\begin{array}{l}\text { Enfants (6-24 mois) au } \\
\text { sein plus aliment de } \\
\text { complément }\end{array}$ & 36,9 & 20,1 & 28,1 \\
\hline $\begin{array}{l}\text { Enfants (24-59 mois) } \\
\text { nourris aux aliments } \\
\text { solides }\end{array}$ & 43,2 & 38,0 & 34,4 \\
\hline
\end{tabular}

Source : données PMSAN, $1^{\text {er }}$ trimestre 2018

Les enfants de 0 à 6 mois nourris au sein sont moins malnutris que les enfants de 6 à 24 mois nourris au sein mais avec un complément alimentaire liquide et/ou solide. Les enfants de 24 à 59 mois sevrés nourris aux aliments solides sont plus astreints à la malnutrition. La prévalence des trois types de malnutrition est plus forte avec l'introduction des aliments de complément ou aliments solides dans l'alimentation du jeune enfant. Ces résultats montrent que le sevrage et la complémentation alimentaire à partir de 6 mois se font très mal. On peut aussi l'expliquer par l'inexistence des offres adéquates et pérennes de services nutritionnels et la faible couverture de l'offre des services de santé au niveau local se traduisant par une faible proportion de population ayant accès à un service de santé.

\subsubsection{Malnutrition liée à l'état de santé de l'enfant}

La maladie et la malnutrition sont fortement corrélées, les deux s'influencent mutuellement. La maladie peut exacerber la malnutrition et vice versa (Tableau 5).

Tableau 5: Les taux de malnutrition (\%) selon une situation de maladie ou non de l'enfant

\begin{tabular}{llll}
\hline Situation de maladie & \multicolumn{3}{c}{ Type de malnutrition (taux \%) } \\
\cline { 2 - 4 } & Taille/ Age <-2ET & Poids/Age <-2ET & Poids/Taille <-2ET \\
\hline $\begin{array}{c}\text { Cas de maladie } \\
\text { (Infection, Diarrhée, } \\
\text { fièvre, etc.) }\end{array}$ & 38,5 & 47,1 & 32,9 \\
\hline Pas de maladie & 36,0 & & \\
\hline Ensemble & 37,2 & 31,8 & 15,8 \\
\hline $\begin{array}{c}\text { Significativité. } \\
\text { p=probabilité du Chi2 }\end{array}$ & 0,000 & 39,4 & 24,3 \\
\hline
\end{tabular}

Source : données PMSAN, ${ }^{\text {er }}$ trimestre 2018

Les taux de malnutrition restent très élevés pour les enfants qui auraient souffert de maladie dans un passé récent comparativement aux enfants qui n'ont pas été malades. Lorsque nous prenons la malnutrition aiguë (Poids/taille), le taux est de 32,9\% pour les enfants ayant souffert de maladie 
dans un passé récent alors que ce même taux est de $15,8 \%$ pour les enfants qui n'ont pas été malades. Les interrelations de cause à effet entre les maladies de l'enfant et la malnutrition sont multiples et interdépendantes. Black et al. (2008) avaient déjà relevé le facteur déterminant de la maladie diarrhéique sur la multiplication des risques de la sous-nutrition des enfants avant 24 mois, avec $25 \%$ de risque de retard de croissance à partir de cinq épisodes de diarrhée chez l'enfant. L'Unicef (1998) retient cinq maladies qui ont un effet direct sur les risques de malnutrition de l'enfant à savoir la diarrhée, la rougeole, les infections respiratoires aiguës, les maladies intestinales d'origine fécale, l'anémie chez l'enfant. Celles-ci constituent les causes immédiates directes de la malnutrition des enfants. On pourrait bien admettre que le contrôle de la malnutrition dans la commune pourrait se faire par un bon contrôle de ces maladies.

\subsection{Déterminants de la malnutrition liés aux caractéristiques familiales}

Certaines causes de la malnutrition sont profondément ancrées dans le fonctionnement des familles et ménages et se traduisent par des variables comme l'âge et l'état nutritionnel de la mère, son niveau d'instruction, son activité, la taille du ménage, les mauvaises habitudes alimentaires, etc.

\subsubsection{Malnutrition liée à l'âge de la mère}

L'âge de la mère influence assez souvent l'état nutritionnel de l'enfant. Le tableau 6 donne la répartition des niveaux de malnutrition des enfants de moins de 5 ans selon l'âge de la mère.

Tableau 6 : Répartition des niveaux de malnutrition des enfants de moins de 5 ans, selon

l'âge de la mère

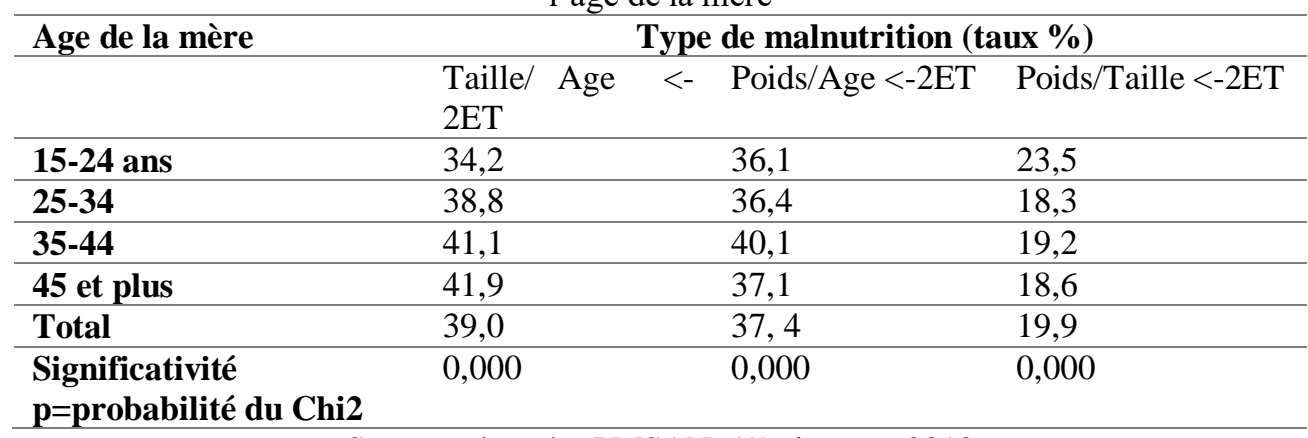

Source : données PMSAN, $1^{\text {er }}$ trimestre 2018

A travers le tableau, on constate que le taux d'émaciation (Poids/Taille $<-2 E T$ ) est assez élevé 23,5\% chez les jeunes mères (15 à 24 ans). Ce taux est un peu plus faible moins de $19 \%$ chez les mères plus âgés ( 25 ans et plus). Par contre les taux des malnutritions retard de croissance (Taille/Age <-2ET) et insuffisance pondérale (Poids/Age <-2ET) sont plus faibles $34,3 \%$ et 36,1 
$\%$ chez les jeunes mères mais élevés plus de $40 \%$ et $37,1 \%$ chez les mères plus âgées ( 25 ans et plus). Elle est à l'inverse plus élevée chez les enfants dont les mères sont plus jeunes et tend à diminuer quand l'âge de la mère augmente. Ces résultats peuvent être expliqués par le fait que la malnutrition retard de croissance s'installe avec le temps et assez souvent suite à des malnutritions aigües mal soignées. Les jeunes mères elles-mêmes, dans une situation de dénutrition, mettent au monde des enfants à petit poids à la naissance (enfants de moins 2,5 kg à la naissance). Compte tenu de leurs expériences limitées et parfois de leur manque d'instruction, les jeunes mères maîtrisent peu l'association des services de soins de santé et des services nutritionnels pour le jeune enfant.

\subsubsection{Malnutrition et niveau d'instruction de la mère}

Le niveau d'instruction de la mère est une des variables qui déterminent la prévalence de la malnutrition chez l'enfant de moins de cinq ans. Plus l'instruction de la mère est élevée, plus sa connaissance en nutrition est bonne et peut apporter à son enfant les soins essentiels en nutrition. Le tableau 7 montre que les niveaux de malnutrition des enfants sont d'autant plus bas que le niveau d'instruction de la mère est élevé.

Tableau 7: Les taux de malnutrition des enfants de moins de 5 ans selon le niveau d'instruction de la mère

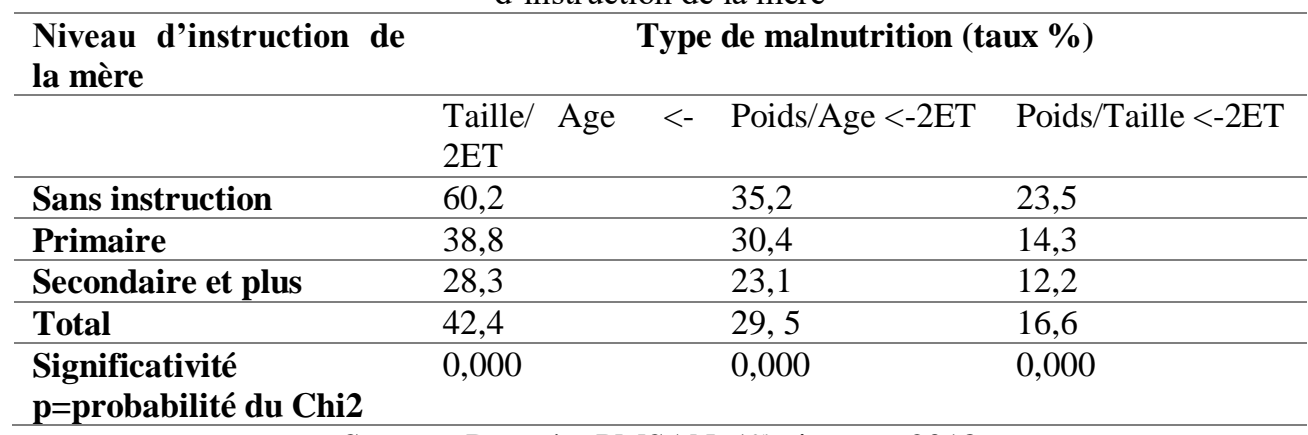

Source : Données PMSAN, $1^{\text {er }}$ trimestre 2018

Pour les trois différents types de malnutrition, les taux sont élevés chez les mères sans instruction, moyennement élevés chez celles qui ont le niveau primaire et bas pour les mères qui ont au moins le niveau secondaire. On constate une forte corrélation du niveau d'instruction de la mère et de la malnutrition chez les enfants. Helen Moestrue reconnaissait déjà que « la nutrition de l'enfant est positivement associée à l'éducation des mères, des pères et grand-mères » (H. Moestue, 2005, page 5). C'est en s'inscrivant dans la même logique que Robert Ndamobissi, (2017) affirmait qu' " en Afrique, le niveau de connaissances de la mère est un vecteur important de socialisation et de changements vers des pratiques de soins modernes et d'alimentation adéquate des enfants ». Ainsi, la faiblesse des connaissances des mères et des 
parents du fait de leur faible niveau d'éducation ainsi que la prédominance des croyances sont autant de facteurs déterminants de la mortalité des enfants (Basu \& Stephenson, 2005 cité par R. Ndamobissi, 2017) et de sous-nutrition des enfants.

\subsubsection{Malnutrition des enfants liée à l'IMC de la mère}

L'indice de masse corporelle (IMC) de la mère est un des indicateurs auxquels on a recourt pour expliquer les causes de la malnutrition chez l'enfant. D'après la figure1, la prévalence de la malnutrition est forte chez les enfants dont l'IMC de la mère est faible.

Figure 1 : Niveaux de malnutrition des enfants de moins de cinq ans par rapport à l'IMC de la mère

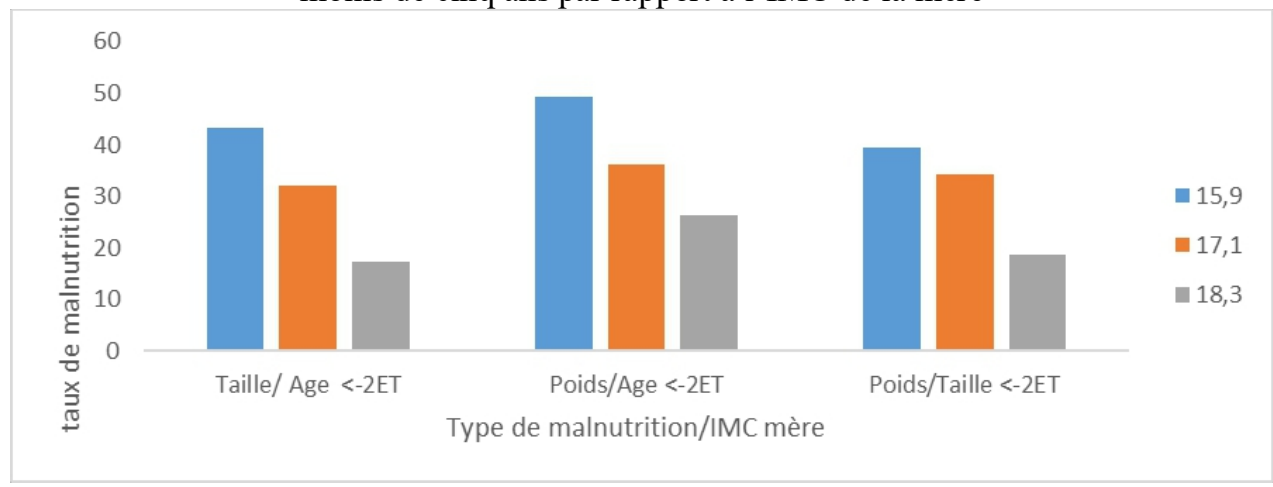

Source : données PMSAN, $1^{\text {er }}$ trimestre 2018 p $<0,000$

Les niveaux de malnutrition des enfants sont élevés pour les mères dont l'IMC est inférieur au seuil de $18,5 \mathrm{Kg} / \mathrm{m}^{2}$. On peut expliquer la relation positive entre la malnutrition de l'enfant et l'IMC de la mère par le fait que le degré d'insuffisance pondérale de la mère l'expose aux risques d'avoir un enfant de faible poids à la naissance. Dans un environnement de pénurie alimentaire permanente et de maladies endémiques comme Karimama, les enfants ayant des mères souffrant de carence alimentaire et nutritionnelle, seraient davantage fragilisés comparativement aux enfants issus des mères bien nourries. Des problèmes d'anémie, souvent cumulées durant l'enfance, l'adolescence et pendant la grossesse chez la femme, sont aussi à l'origine de la malnutrition et du faible poids à la naissance de l'enfant.

\subsubsection{Malnutrition liée à l'activité de la mère}

L'occupation de la mère peut être bénéfique pour l'état nutritionnel de l'enfant comme cela peut avoir des effets négatifs. Dans la commune, on remarque que l'occupation de la mère a tendance à impacter négativement l'état nutritionnel de l'enfant (tableau 8). 
Tableau 8 : Taux de malnutrition des enfants selon que la mère est occupée par un travail ou pas

\begin{tabular}{|c|c|c|c|}
\hline \multirow{2}{*}{$\begin{array}{c}\text { Mère occupée par un } \\
\text { travail }\end{array}$} & \multicolumn{3}{|c|}{ Type de malnutrition (taux \%) } \\
\hline & $\begin{array}{l}\text { Taille/ Age } \\
\text { 2ET }\end{array}$ & $<-\quad$ Poids/Age $<-2$ ET & Poids/Taille $<-2$ ET \\
\hline Oui & 36,2 & 23,2 & 18,5 \\
\hline Non & 38,8 & 23,6 & 16,3 \\
\hline Total & 35,2 & 22,7 & 15,9 \\
\hline $\begin{array}{l}\text { Significativité } \\
\text { p=probabilité du Chi2 }\end{array}$ & 0,000 & 0,000 & 0,000 \\
\hline
\end{tabular}

Source : Données PMSAN, $1^{\text {er }}$ trimestre 2018

L'occupation de la mère par une activité a un effet réel sur la malnutrition des enfants. Le taux du retard de croissance est plus faible $(36,2 \%)$ chez les enfants dont les mères ont une occupation et plus élevé $(38,8$ $\%$ ) chez les enfants dont les mères sont sans occupation. Mais le contraire est observé au niveau de l'émaciation, où les enfants des mères occupées par un travail sont plus affectés $(18,5 \%)$ contre $16,3 \%$ pour les enfants dont les mères ne travaillent pas. En ce qui concerne la malnutrition insuffisance pondérale, la différence n'est pas sensible. On peut comprendre cela par le fait que les femmes qui travaillent, très occupées par leur travail, ont tendance à négliger les soins quotidiens à apporter pour l'alimentation de leurs enfants. Mais elles en prennent soins dès qu'elles remarquent une situation d'anomalie chez l'enfant parce qu'elles ont les moyens de le faire.

\subsubsection{Malnutrition liée à la taille du ménage}

Le risque d'insécurité alimentaire est plus grand pour des ménages qui ont une grande taille. L'insécurité alimentaire du ménage expose les enfants et se traduit par la malnutrition de ces êtres très vulnérables (figure 2).

Figure 2 : Taux de malnutrition des enfants de moins de cinq ans selon la taille du ménage

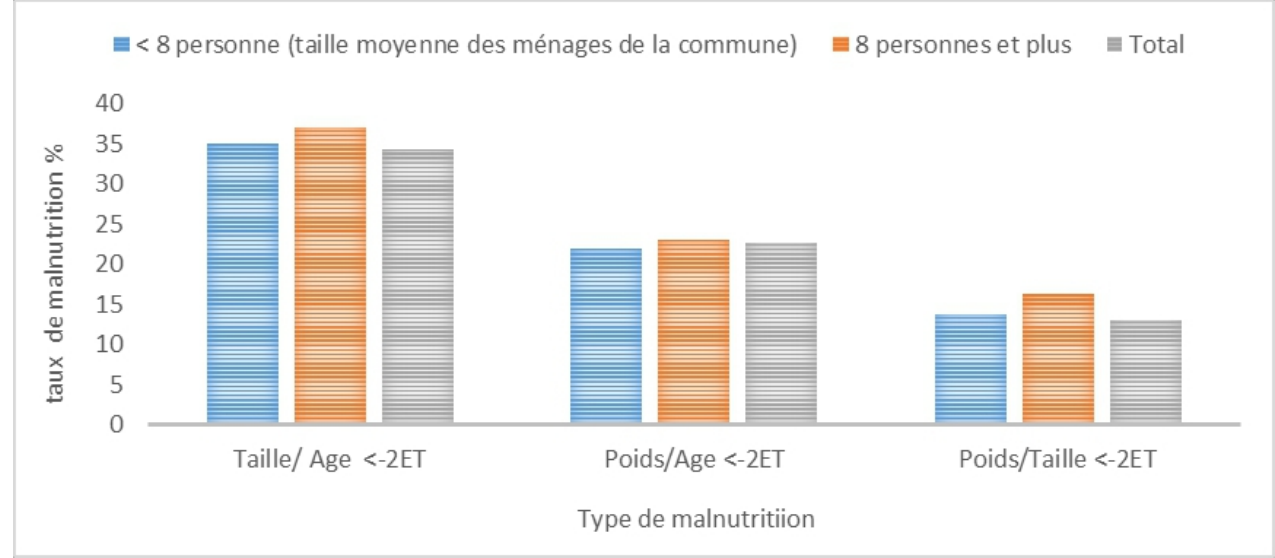

Source : Données PMSAN, $1^{\text {er }}$ trimestre $2018 \quad \mathrm{p}<0,010$ 
Les niveaux de malnutrition sont plus faibles pour les ménages de plus petite taille, mais ils augment avec les ménages à effectif plus important. Dans un milieu comme Karimama où le score de consommation alimentaire est très faible, la ration alimentaire inadéquate et la diversification alimentaire pauvre, cette influence de la taille du ménage sur la malnutrition des enfants se présente comme une évidence. Dans la commune de Karimama, on a remarqué une faible utilisation du bol individuel pour l'enfant, une consommation journalière à base de céréales (bouillies de céréales), une absence de privilège pour les enfants qui mangent dans un plat commun; parfois la satisfaction de l'époux prime sur celle des enfants et enfin un effort limité fourni par la mère pour que les repas conviennent à l'enfant.

\subsubsection{Malnutrition liée à la durée de l'intervalle inter génésique précédent et à la pratique contraceptive de la mère}

L'intervalle inter génésique précédent et la pratique contraceptive ont un effet considérable sur la malnutrition des enfants de moins de cinq ans (figure 3 ).

Figure 3 : Taux de malnutrition des enfants selon la durée de l'intervalle inter génésique précédent et à la pratique contraceptive de la mère

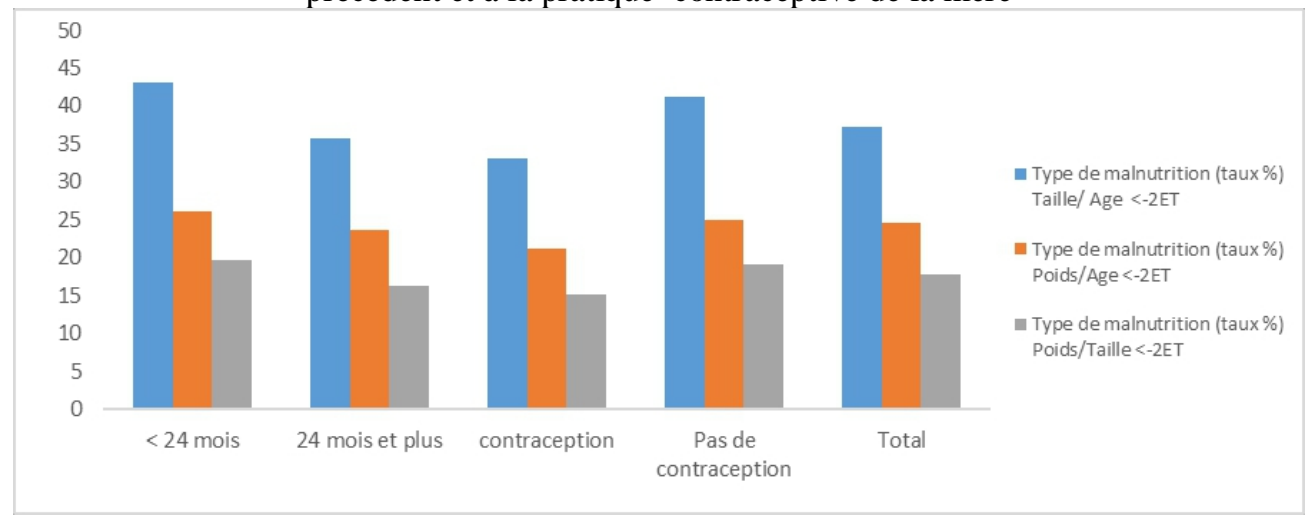

Source : Données PMSAN, $1^{\text {er }}$ trimestre 2018

Les niveaux de malnutrition selon l'intervalle inter génésique précédent baissent au fur et à mesure que celui-ci s'allonge. En tant qu'un facteur d'allongement de l'intervalle inter génésique précédent ou un facteur de limitation des naissances, la pratique contraceptive a le même effet que celui-ci. Les enfants des mères qui pratiquent la contraception, même traditionnelle, ont des niveaux de malnutrition plus faibles que ceux des mères qui ne le font pas. En effet, un autre enfant qui naît dans le ménage mobilise plus l'attention des parents et peut amener le grand frère à avoir ses soins nutritionnels réduits ou carrément les deux enfants peuvent en partir. La mère pour avoir eu des accouchements rapprochés peut mettre en péril sa santé, ce qui aurait un effet négatif sur l'état nutritionnel des enfants. 


\subsection{Déterminants de la malnutrition liés aux caractéristiques communautaires}

Les caractéristiques communautaires favorisant la malnutrition des enfants de moins de cinq ans seront appréciées à travers des variables de résidence, de pauvreté du ménage, de l'accès à l'eau potable et à l'hygiène.

\subsubsection{Malnutrition et milieu de résidence}

Dans la commune de Karimama, la malnutrition est plus prégnante dans les arrondissements un peu plus reculés (Monsey Haoussa, Kompa, Bogobogo, Gouroukambou, etc.). La prévalence est plus faible dans l'arrondissement de karimama centre où la population est relativement plus urbanisée. La prévalence du retard de croissance atteint plus de 45,0 \% chez les enfants de moins de 5 ans dans les villages éloignés contre $39 \%$ chez les enfants vivant au centre de Karimama. Les deux autres types de malnutrition (l'émaciation et l'insuffisance pondérale) sont également plus remarqués en milieu rural qu'en milieu urbain chez les enfants âgés de moins de 5 ans. L'éloignement des villages et leur accès peu facile ne facilitent pas l'information dans le domaine de l'éducation nutritionnelle. Les difficultés d'accès des villages aux soins adéquats de santé peuvent aussi expliquer l'influence du facteur milieu résidence dans la nutrition.

\subsubsection{Malnutrition et pauvreté du ménage}

La malnutrition est liée aux indicateurs macro-économiques, aux variables de santé et à l'état nutritionnel des mères. Les niveaux des prévalences de malnutrition observées dans la commune de Karimama sont très sensibles à leurs interactions. Les prévalences de malnutrition sont plus élevées chez les enfants des ménages pauvres que chez ceux issus de ménages riches ou aisés. La prévalence du retard de croissance atteint parfois $51 \% \mathrm{chez}$ les enfants de moins de 5 ans des ménages très pauvres contre $28 \%$ chez les enfants issus de ménages très riches. Le même constat se fait avec la prévalence de la maigreur chez les enfants de moins de 5 ans qui est plus élevée dans des ménages pauvres et faible chez les enfants des ménages riches. Quant à l'insuffisance pondérale, sa prévalence atteint 18,2 \% chez les enfants des ménages très pauvres contre $14,1 \%$ chez les enfants des ménages très riches. Le revenu a une influence sur la malnutrition à travers la disponibilité alimentaire et l'environnement sanitaire. En effet, plus le revenu croit, plus on est à même de se procurer les aliments indispensables à la ration alimentaire. De la même manière, lorsque l'on dispose des ressources nécessaires, on peut recourir normalement et dans un délai raisonnable aux soins de santé ; ce qui évite une destruction précoce du métabolisme. Cette destruction si elle intervenait, elle peut conduire à un manque d'appétit et donc à une 
augmentation du taux de prévalence de la malnutrition. On comprend pourquoi la richesse des ménages est le prédicteur le plus puissant de la malnutrition des enfants » (J-C. Fotso et B. Kuate-Defo, 2005, p16-17). Ainsi, le faible revenu participe de la pauvreté des ménages, qui lui-même affecte l'état nutritionnel des enfants. La pauvreté en limitant la capacité productive des ménages, affecte le développement de l'ensemble de la communauté. Dans une étude portant sur la relation entre l'insécurité alimentaire du ménage et l'état nutritionnel des enfants de 0 à 23 mois en milieu urbain au Kenya, M. Mutisya et al. (2015) avaient indiqué les risques de retard de croissance qui augmentent de $12 \%$ chez les enfants vivant dans un ménage en état d'insécurité alimentaire.

\subsubsection{Malnutrition source d'eau et hygiène du ménage}

Les enfants vivant dans les ménages disposant de moyens d'assainissement sont moins touchés par les trois types de malnutrition que les enfants des ménages ne disposant pas de moyens d'assainissement. Dans la commune de Karimama, la prévalence du retard de croissance atteint $43 \%$ chez les enfants des ménages ne disposant pas de moyens d'assainissement contre $35 \%$ chez les enfants vivant dans les ménages disposant de moyens d'assainissement. Les prévalences des deux autres types de malnutrition (émaciation /maigreur, insuffisance pondérale) sont aussi plus élevées chez les enfants de moins de 5 ans vivant dans les ménages ne disposant pas de moyens d'assainissement que chez les enfants des ménages disposant de moyens d'assainissement. La prévalence du retard de croissance est très élevé et varie de $52 \%$ chez les enfants dont les ménages consommant l'eau provenant des sources comme la rivière, les marres à $39 \%$ là où ils consommant l'eau de puits protégés. Les enfants des ménages ayant accès à l'eau de forage souffrent moins de malnutrition (26\%). L'accès de la population à l'eau potable pour la boisson et l'utilisation des latrines/toilettes adéquates ou protégées reste encore un défi majeur pour la commune. Ces résultats rejoignent W.H.O, (2000) pour qui la malnutrition affecte tous les groupes d'âge, mais elle est particulièrement fréquente chez les sujets à faible revenu, avec un accès insuffisant à l'eau potable et privés d'éducation sanitaire satisfaisante.

\subsection{Déterminants de la malnutrition liés aux facteurs socioculturels}

Les facteurs socioculturels constitués des normes et valeurs d'une communauté sont liées à l'environnement de l'individu et influencent négativement ou positivement l'alimentation et les soins de santé des jeunes enfants. Les perceptions, les croyances, les normes sociales et les valeurs communautaires véhiculées au sein de la commune sont des facteurs qui déterminent l'état nutritionnel de l'enfant. 


\subsubsection{Perceptions des causes de la malnutrition des enfants}

L'insuffisance de connaissances de la malnutrition des enfants et de ses causes par les ménages ruraux est un facteur handicapant son éradication. Dans la commune de Karimama, la malnutrition est assez souvent associée à une maladie comme tant d'autres, un sort divin, un résultat de l'infidélité de la femme ou parfois une punition des dieux. Ces considérations liées à la perception du mal renforcent la sévérité des cas de malnutrition aiguë des enfants et exposent davantage les enfants affectés aux risques de décès. Les mauvaises perceptions de l'état morbide de la malnutrition des enfants à cause de l'ignorance ou des croyances collectives, constituent un facteur de risque très aggravant pour le recours aux soins et la survie des enfants. Dans les études de facteurs déterminants de la malnutrition, de la santé ou de la mortalité des enfants en Afrique, il a été prouvé l'impact des croyances, des coutumes ou des principes traditionnels sur la demande des services de santé ou de nutrition ainsi que sur les attitudes ou les pratiques de soins de santé et nutritionnels apportés aux enfants (E.M. Akoto, 1993). En effet, la sousnutrition modérée n'est pas généralement reconnue comme un problème de santé nécessitant un traitement, mais plutôt comme une situation de routine chez l'enfant et gérable avec la fausse perception relative à la perte de poids typiquement saisonnière (M. Muraya et al. 2016). Il affirmait que dans les cas où les symptômes sont considérés comme trop sérieux et exigeant des mesures correctives, les stratégies de gestion du ménage étaient généralement fondées sur les perceptions erronées de l'étiologie de la maladie (par exemple, l'enfant est malade à cause de l'acte d'infidélité sexuelle de son père ou de sa mère dans le village).

\subsubsection{Croyances et pratiques du mariage précoce et forcé des femmes}

Les normes socioculturelles dictées par des perceptions, l'appartenance ethnique ou des croyances religieuses impactent beaucoup l'alimentation de l'enfant. Le tableau 9 présente les pratiques de mariage liées aux croyances religieuses dans la commune.

Tableau 9: Evolution du nombre d'enfants victimes de mariage précoce

\begin{tabular}{llllllll}
\hline Année & $\mathbf{2 0 1 3}$ & $\mathbf{2 0 1 4}$ & $\mathbf{2 0 1 5}$ & $\mathbf{2 0 1 6}$ & $\mathbf{2 0 1 7}$ & $\mathbf{2 0 1 8}$ & Total \\
\hline $\begin{array}{l}\text { Nombre d'enfants (filles) victimes de } \\
\text { mariage précoce }\end{array}$ & 21 & 19 & 18 & 17 & 14 & 12 & 101 \\
\hline $\begin{array}{l}\text { Nombre de femmes victimes de } \\
\text { mariage forcé }\end{array}$ & 29 & 22 & 21 & 23 & 16 & 14 & 125 \\
\hline
\end{tabular}

Source : Données Centre de Promotion Sociale (CPS), 2018

Dans un intervalle de 5 ans (2013 et 2018), le nombre d'enfants victimes de mariage précoce est de 101 et le nombre de femmes poussés au mariage forcé est de 125. On comprend que dans la commune de Karimama, le mariage 
précoce de jeunes filles est une pratique encrée dans la culture. Cette pratique a pour sous bassement la croyance religieuse qui tirerait sa source de la foi islamique. Les filles sont souvent mariées à partir de l'âge de12 ans. Pour justifier cette pratique, les leaders religieux évoquent des recommandations religieuses inspirées des lois fondamentales comme le coran. Ces recommandations se traduisent entre autres par des expressions :

- $\quad$ "C'est une malédiction pour une jeune fille d'avoir trois règles sur le toit de ses parents"

- $\quad$ "Il faut marier vite la jeune fille avant qu'elle ne perde sa virginité et ne naisse en elle l'instinct de prostitution".

- "Lorsqu'il s'agit du mariage, les filles n'ont pas de volonté car les parents savent mieux ce qu'il leur faut."

Les mariages précoces et forcés de femmes renforcent la violence sexuelle basée sur le genre et ont un impact négatif sur l'égalité de genre et l'autonomisation des femmes et des filles. La santé des mères, des nouveaunés et des enfants n'est pas non plus épargnée ; ce qui d'ailleurs a un effet direct sur la nutrition des jeunes enfants. Lorsqu'on sait que les améliorations significatives et durables dans la nutrition sont dues à une combinaison d'actions spécifiques, on peut affirmer sans risque de se tromper que les mariages précoces et forcés sont des facteurs déterminants des taux élevés de la malnutrition dans la commune. Au Zimbabwe, d'après une étude de l'UNICEF, réalisée en 2011, les croyances radicales des sectes religieuses dénommées « sectes apostoliques d'origine africaine » qui rejettent l'utilisation des services modernes de santé et d'éducation, ont eu un impact très négatif sur les indicateurs sociaux des enfants appartenant à ces groupes religieux (UNICEF, 2011).

\subsubsection{Discrimination sexuelle et absence de pouvoir décisionnel des femmes}

Au cours des entretiens, les femmes ont parlé des risques qui prennent directement racine dans les inégalités de genre et les normes culturelles qui dictent ce qu'elles peuvent ou ne peuvent pas faire. La discrimination sexuelle sous toutes ses formes et les stéréotypes sexistes font partie des risques évoqués par la quasi-totalité des femmes (105 sur 125 femmes). Elles ont expliqué que l'absence de pouvoir décisionnel des femmes avait des répercussions directes non seulement sur elles-mêmes, mais sur la capacité à nourrir et à prendre soins de leurs enfants et de leurs ménages. Les femmes ont précisé que le statut social inférieur des femmes les empêche souvent d'utiliser leurs connaissances propres sur la nutrition et l'alimentation des enfants et les soins de santé. En ce qui concerne, le pouvoir de décision, la plupart des décisions sont prises dans le ménage par les hommes, souvent 
beaucoup plus âgés que leurs femmes. Par exemple, pour une décision aussi importante que la gestion des réserves alimentaires de la famille, l'avis de la femme compte très peu. Le risque de la malnutrition devient élevé lorsqu'il arrive que le chef de famille pour des besoins d'argent vide le grenier. En effet, l'homme quand il se sent démuni vend une bonne quantité de la réserve alimentaire du ménage quitte à exposer son ménage. Les femmes sont plus vulnérables aux risques en raison des pratiques traditionnelles existantes qui ne considèrent pas les femmes comme étant égales aux hommes. Les femmes par exemple n'héritent pas de terre, elles ne peuvent pas produire certaines spéculations (l'igname par exemple), elles sont limitées par la division sexiste du travail, la discrimination en matière de droits fonciers. L'indépendance de la femme lui donnera la latitude d'opérer des choix et de prendre des décisions en faveur de son ménage et surtout de l'alimentation des enfants.

\subsubsection{Violence faite aux femmes et forte maternité}

La violence faite aux femmes et la forte maternité dans la commune sont parmi les risques de malnutrition évoqués par les femmes. Plus de $98 \%$ des femmes et hommes enquêtés affirment que les risques liés aux pratiques traditionnelles telles que la mutilation génitale féminine, le viol et le mariage précoce prennent naissance au sein même des ménages. Bien que l'on donne peu d'importance à ces risques, ils ont tendance à exacerber la malnutrition. Ils nuisent énormément à la nutrition par le biais des soins de santé et nutritionnels insuffisants associés à une faible d'attention accordé à l'enfant. En ce qui concerne la forte maternité, dans la commune de Karimama, le nombre moyen d'enfants par ménage est de sept (07) et quatre (04) femmes sur cinq (05) portent d'enfants. Le délai d'attente avant d'avoir un prochain enfant est en moyenne d'un an. Ces facteurs expliquent la pratique d'une maternité très active dans la commune. Ce phénomène qui mette en mal la santé physiologique des mères réduit la qualité de l'alimentation de l'enfant. En effet, les femmes reconnaissent que la naissance d'un nouvel enfant exerce une grande pression sur la sécurité alimentaire du ménage. Il faut désormais augmenter le disponible vivrier dans le ménage et aussi nourrir des parents venus d'ailleurs pour porter main forte à la nouvelle maman. Le risque de malnutrition des enfants nés de mères d'un jeune âge et d'une naissance rapprochée est plus élevé du fait de l'inexpérience et de l'état physiologique faible de cette dernière.

\subsubsection{Travail non rémunéré des femmes}

Plus de $95 \%$ des femmes enquêtées affirment travailler beaucoup trop pour avoir le temps de supprimer totalement les risques de malnutrition de leurs enfants. Le même constat est fait par les hommes (75\%) qui reconnaissent le travail invisible et la responsabilité disproportionnée des 
femmes en matière de soins non-rémunérés. Ce travail un peu à l'excès chez les femmes, qui se traduit par les travaux domestiques, les soins donnés aux enfants, l'aide apportée aux champs à son mari et sa responsabilité à prendre soin de toute la famille, les prive de temps pour participer à des entreprises productives et aux espaces décisionnels. Pourtant dans cette perception inappropriée du rapport genre, la femme est considérée comme responsable première de la mauvaise santé ou de la sous-nutrition des enfants au détriment de l'homme » (M. Muraya \& al, 2016, p10).

\section{Discussion}

Les résultats obtenus dans cette étude montrent une relation significative entre la malnutrition des enfants et les paramètres individuels, familiaux et environnementaux. Ces paramètres associés aux déterminants socioculturels constituent des variables contextuelles influençant à la fois l'émaciation, l'insuffisance pondérale et le retard de croissance. Dans les pratiques de soins aux enfants malnutris dans la commune, il est souvent privilégié les approches facilitant l'établissement des facteurs corrélés avec les comportements alimentaires, en lien notamment avec les caractéristiques psychologiques individuelles des enfants. En effet, «de nombreuses interventions dans le domaine de la nutrition s'appuient sur des théories fondées sur les caractéristiques psychologiques de l'individu, comme ses attitudes ou son sentiment d'efficacité personnelle (en anglais, self-efficacy), c'est̀-à-dire les croyances qu'un individu a dans ses propres capacités d'action» (A. Bandura, 1977, p.201). Le Rapport global nutrition 2016 reconnaît que " la pauvreté et les inégalités, l'eau, l'assainissement et l'hygiène, l'éducation, les systèmes alimentaires, le changement climatique, la protection sociale et l'agriculture, tous ont un impact important sur les résultats de la nutrition » (IIRPA, 2016, p6). C'est dans la même logique que la Déclaration de Rome sur la nutrition a identifiés les défis de la malnutrition que sont: (i) la pauvreté, le sous-développement et le bas niveau socioéconomique; (ii) le manque d'accès permanent à une nourriture suffisante ; (iii) les mauvaises pratiques alimentaires et de soin des nourrissons et des jeunes enfants, les conditions sanitaires et d'hygiène médiocres, l'accès insuffisant à l'éducation, etc. ; (iv) les épidémies (FAO, OMS, 2014). Ces facteurs mettent plus l'accent sur le facteur "humain" et "individu" de la malnutrition et relèvent peu l'imbrication de l'individu au sein de différentes structures sociales auxquelles il appartient, qui pourtant conditionnement son action de par les faits sociaux et culturels. Les comportements individuels sont fortement dictés par les codes collectifs qui impactent l'état nutritionnel des enfants. J.P. Poulain dira que «...les pratiques alimentaires sont des marqueurs identitaires et par lesquelles se déploient des codes de différenciation sociale... » (J.P. Poulain, 2002, p137). Il est ainsi nécessaire 
de traiter le problème de malnutrition dans tous ses aspects (K. Klennert (éd.), 2006, p120). Outre les facteurs relatifs au travail des femmes et à leur contrôle sur les ressources, les facteurs socioculturels que constituent les croyances et les coutumes exercent également une influence considérable sur le bien-être nutritionnel des familles. «L'alimentation est à la fois hors et dans le fait social en ce sens que les mœurs alimentaires préexistent aux individus, mais que l'alimentation est plus biologique que sociologique avec pour conséquence, l'alimentation devient un lieu d'indexation de problématiques sociologiques plus fortes » (J.P. Poulain, 2002, p212). On ne saurait penser normes nutritionnelles et lutte contre la malnutrition dans l'ignorance totale des normes sociales. La culture est un facteur puissant du comportement qui conditionne la pratique de l'alimentation et de la nutrition. Certaines croyances, habitudes et pratiques d'origine culturelle peuvent nuire ainsi à la nutrition. Pour une mauvaise perception communautaire du rapport genre, les femmes sont considérées comme responsable première de la mauvaise santé ou de la sous-nutrition des enfants au détriment de l'homme (M. Muraya \& al, 2016, p10). A Karimama, les influences socioculturelles concernent les systèmes coutumiers de mariages précoces et forcés, les violences faites aux femmes, une fécondité forte et rapproché, les facteurs religieux, la discrimination de tout genre. Ils pèsent lourdement sur la nutrition. Pour réduire cette forme de malnutrition dans la commune, il est indispensable de faire mieux comprendre les antécédents sociaux et culturels des coutumes qui se révèlent dangereuses pour la nutrition. Ces facteurs qui ne sont souvent pas associés aux causes profondes et fondamentales de la malnutrition ont une influence importante sur la nutrition dans la commune. Ils constituent même la racine de la malnutrition chronique des enfants. Pour exemple, il est constaté dans la commune que les enfants nés de mères très jeunes, qui n'ont pas terminé elles-mêmes leur croissance, deviennent très fréquemment malnutris. Si reculer l'âge de la première grossesse à Karimama où traditionnellement les filles sont mariées très jeunes, à partir de 12 ans, est hautement souhaitable pour donner aux mères la possibilité d'espacer les naissances, il est aussi essentiel pour prévenir la malnutrition. Mais le faire peut rencontrer des obstacles rédhibitoires des leaders religieux. Ainsi, les mesures liées à retarder l'âge à partir duquel une femme commence à avoir des enfants et à espacer le plus possible les naissances, l'allégement du fardeau de la reproduction ou l'amélioration de la place de la femme dans la société ne peuvent se faire qu'en modifiant les attitudes et pratiques culturelles en faveur de l'égalité des femmes. L'univers de valeurs des groupes sociaux représente une richesse certaine mais peut-être, sans qu'on ne s'en rende compte, une contrainte majeure pour l'état nutritionnel des enfants. Dans leur étude réalisée en 2005 portant sur cinq pays d'Afrique (Burkina Faso, Cameroun, Égypte, Kenya, Zimbabwe), J-C Fotso et B. Kuate-Defo ont fait un lien entre les inégalités 
socio-économiques, sociales, communautaires et familiales et le statut nutritionnel des enfants. Ils ont montré que les pratiques nutritionnelles et alimentaires entretiennent un lien étroit avec les pratiques culturelles et les représentations sociales.

\section{Conclusion}

L'examen des facteurs déterminants la malnutrition des enfants, à savoir l'accès des ménages à la nourriture; les soins aux mères et enfants et les pratiques d'alimentation ; la salubrité de l'environnement et l'accès à la santé montre que la malnutrition est le résultat d'une combinaison de plusieurs facteurs. Dans la complexité de l'analyse de ces facteurs réside un fort poids $\mathrm{du}$ facteur humain, social et culturel aussi bien dans les causes perçues que dans les solutions envisagées. Un examen approfondi du rôle et des responsabilités des femmes en ce qui concerne le bien-être nutritionnel de la famille nécessite de prendre en compte le contexte social pour mieux comprendre la malnutrition dans la Commune de Karimama. Les croyances, le contexte socioculturel et les risques auxquels les femmes sont tout particulièrement confrontées ont une grande influence sur la nutrition. Les mariages précoces et forcés, la discrimination sexuelle des femmes, la répartition sexiste du travail, l'exode rural des hommes, la violence contre les femmes, l'absence de pouvoir décisionnel des femmes ne sont pas souvent directement lié à la nutrition pourtant ils constituent la racine de la malnutrition. Par l'influence et les pressions qu'ils exercent sur l'alimentation des femmes, des soins de santé des enfants et de la famille, ces facteurs font le nid à la malnutrition chronique. Mais la prise en compte de tous ces paramètres dans les pratiques de la nutrition suffirait-elle pour éradiquer la malnutrition ? Y aurait-il des mécanismes de décision liés à l'environnement socioculturel qui impacterait plus positivement la nutrition dans cette Commune ?

\section{References:}

1. Akoto E.M., (1993), Déterminants socioculturels de la mortalité des enfants en Afrique Noire, Hypothèses et recherche d'explication, Louvain la Neuve, Académia.

2. Akoto E.M et Allan G. Hill, K. (1988). « Morbidité, malnutrition et mortalité des enfants », in «Population et Sociétés en Afrique au sud du Sahara ».Ed par D. Tabutin, Paris Harmattan, pp 309-334.

3. Allomasso, R., Coulibaly, O., Glitho, I., Hell, K. (2006). Principaux facteurs affectant l'état nutritionnel et de santé des enfants exposés à l'aflatoxine au Bénin: Application du model Probit. Bulletin de la Recherche Agronomique du Bénin. Numéro 53 - Septembre 2006, p.917 
4. Ancell T. (2002). Statistiques/Épidémiologie. Paris, Éditions Maloine

5. Bandura, A. (1977). Self-efficacy: Toward a unifying theory of behavioral change. Psychological Review, 84(2), 191-215. http://dx.doi.org/10.1037/0033-295X.84.2.191

6. Basse, MT. (1986). Les aspects socio-culturels et économiques de la nutrition dans les pays en voie de développement. In : Lemonnier D, Ingenbleek Y, eds. Les malnutritions dans les pays du tiers-monde. Paris: INSERM, 633-40.

7. Beaton, G., A. Keily, J- Kevany, R. Martorell and J. (1990) MasonAmropriate uses of anthropometric indices in children, A report based on an ACUSCN workshop. ACUSCFi state-of-the-art series. Nutrition policy discussion papa No 7

8. Bellamy, C. (1998). La situation des enfants dans le monde 1998New-York, UNICEF, 141p.

9. Black. T., \& al. (2008). Maternal and child under nutrition: global and regional exposures and health consequences. (T. Lancet, Ed.) The Lancet $\mathrm{n}^{\circ}$ 9608, series 371, 371. Retrieved 2012

10. Bourdieu, P. (1977). Remarques provisoires sur la perception des corps. Actes de la recherche en sciences sociales, $14: 51-54$

11. Bourdieu, P. (1079). La distinction. Critique sociale du jugement. Collection Le sens commun, éditions de Minuit, Paris

12. Delpeuch, F. et Salem G. (2002). Nutrition publique : nutrition et développement au temps de la mondialisation : de nouveaux enjeux pour la santé publique. Santé : Cahiers d'Etudes et de Recherche Francophones, 12 (1), 118 p. ISSN 1157-5999

13. Dabo, K., Traoré, S., Traoré, B. (2008). Analyse des causes de la malnutrition dans trois pays du Sahel Burkina Faso, Mali et le Tchad, Rapport de Recherche. Ouagadougou: Centre des Etudes et Recherches en Population et Développement - CERPOD - CILSS.

14. FAO. (2012). Rapport sur la situation alimentaire et nutritionnelle mondiale 2012. Rome

15. FAO/OMS. (2014). Conférence internationale sur Ia nutrition. Déclaration mondiale sur la nutrition et plan d'action, Rome,

16. Field, A. (2005). Discovering Statistics using SPSS second edition. London, United Kingdom: Sage Publications Ltd.

17. Fotso, J-C, \& Debo, B. (2005). Household and Community Socio economic influences on early childhood malnutrition in Africa. PRONUSTIC Research Laboratory at the University of Montreal, 22 pages.

18. IIRPA. (2016, vxiii). Rapport Global Nutrition 2016 : de promesse à l'impact effectif, mettre fin à la malnutrition d'ici 2030. Institut International de Recherche sur les Politiques Alimentaires. 
Washington: Institut International de Recherche sur les Politiques Alimentaires.

19. Klennert, K. (éd.) (2006). Assurer la sécurité alimentaire et nutritionnelle. Actions visant à relever le défi global, Manuel de références, Feldafing, Inwent, 260 p.

20. Lachaud, JP. (2005). La dynamique de l'inégalité de la malnutrition des enfants. Une analyse comparative fondée sur une décomposition de régression. Bordeaux - France: Université Montesquieu-Bordeaux V - Document de travail numéro 86.

21. Muraya, M. et al. (2016). Perceptions of childhood undernutrition among rural households on the Kenya Coast - a qualitative study. (B. Central, Éd.) BMC Public Health, 11 p.

22. Ndamobissi, R. (2017). Les défis sociodémographiques et politiques de la malnutrition des enfants dans les pays d'Afrique du Sahel et de la Corne de l'Afrique. Sociologie. Université Bourgogne FrancheComté, Français. NNT : 2017UBFCH029. tel-01793437

23. Nelson, E. et al. (2009) Modeling multiple ecosystem services, biodiversity conservation, commodity production, and tradeoffs at landscape scales Erik Nelson Front Ecol Environ; 7(1): 4-11, doi:10.1890/080023

24. Nestle M, Wing R, Birch L, Disogra L, Drewnowski A, Coll, (1998). Behavioral and social influences on food choice. Nutr Rev, 56: S50S64

25. Olivier de Sardan JP, (1997). Anthropologie et développement. Essai en socio-anthropologie du changement social. Paris: APAD-Karthala; $221 \mathrm{p}$.

26. OMS. 1986a. Guide pour la formation en nutrition des agents de santé communautaire. WHO Offset Publication $n^{\circ} 59$. Genève

27. Pelletier, D.L. (1994). The potentiating effects of malnutrition on child mortalhy : Epidemiologic evidence and poficy implications- Nutr Rev, 52 (12) : 409-415.

28. Poulain, J.P. (2002). Sociologies de l'alimentation, les mangeurs et l'espace social alimentaire (éd. 4e édition de Janvier 2017). (Quadrige, Éd.), Paris, France: Presses Universitaires de France, 212p.

29. Régnier F., Lhuissier A., Gojard S. (2009). Sociologie de l'alimentation. La Découverte, « Repères », 2009, 128 pages. ISBN : 9782707148452. URL : https://www.cairn.info/sociologie-de-1alimentation--9782707148452.htm

30. Sen, A. (1999). Development as Freedom. Oxford, United Kingdom: Oxford University Press.

31. Sen, A. (1981). Poverty and Famines: An Essay on Entitlement and Deprivations. Oxford, Royaume Uni: Oxford University Press, P1-4. 
32. Suremain, CE. (2000). Dynamiques de l'alimentation et socialisation du jeune enfant à Brazzaville (Congo). Autre part; 15: 73-91.

33. UNICEF. (1998). La situation des enfants dans le monde.

34. Victora CG, Adair L, Fall C, Hallal PC, Martorell R, Richter L, Sachdev HS. (2008). Maternal and child undernutrition: consequences for adult health and human capital. Lancet. Jan 26;371(9609):340-57. doi: 10.1016/S0140-6736(07)61692-4.

35. W.H.O., (2000). Turning the tide of malnutrition: responding to the challenge of the 21 st century. Geneva : (WHO/NHD/00.7). 